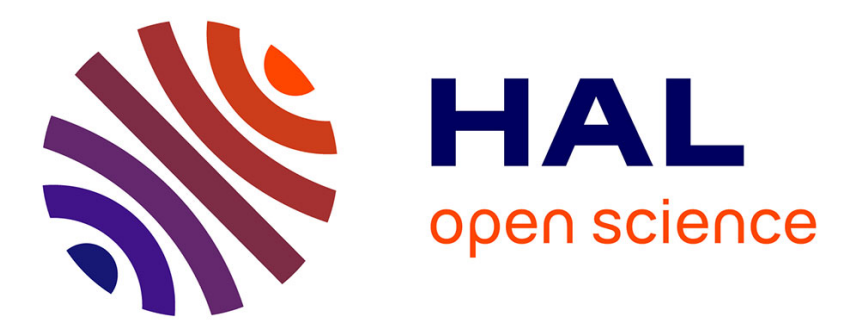

\title{
LE " LACTOTEST " DE KOSCHUSCHAROFF. Le moyen le plus simple pour rechercher l'addition du lait de vache au lait de femme
}

Marie-Elise Kayser

\section{- To cite this version:}

Marie-Elise Kayser. LE " LACTOTEST " DE KOSCHUSCHAROFF. Le moyen le plus simple pour rechercher l'addition du lait de vache au lait de femme. Le Lait, 1938, 18 (179), pp.930-934. hal00895339

\section{HAL Id: hal-00895339 \\ https://hal.science/hal-00895339}

Submitted on 1 Jan 1938

HAL is a multi-disciplinary open access archive for the deposit and dissemination of scientific research documents, whether they are published or not. The documents may come from teaching and research institutions in France or abroad, or from public or private research centers.
L'archive ouverte pluridisciplinaire HAL, est destinée au dépôt et à la diffusion de documents scientifiques de niveau recherche, publiés ou non, émanant des établissements d'enseignement et de recherche français ou étrangers, des laboratoires publics ou privés. 
"Paix à la mémoire de ces aveugles qui, s'ils avaient vaincu, auraient mérité les malédictions de l'humanité, n

En conclusion, le but que nous devons poursuivre, en faveur de l'hygiène du lait de consommation en nature, nous paraît être le même que celui poursuivi ailleurs, dont la réalisation exige partout les mêmes moyens classiques ayant prouvé solidement leur efficacité, nous voulons dire : une vigoureuse inspection complétée par une bonne pasteurisation.

\title{
LE « LACTOTEST » DE KOSCHUSCHAROFF (1)
}

\section{Le moyen le plus simple pour rechercher l'addition du lait de vache au lait de femme}

\author{
par
}

\section{le Dr MaRIe-Elise KAYSER}

de la Clinique régionale pour femmes d'Erfurt.

On sait qu'il a été créé en Allemagne un dépôt central de lait de femme qui recueille le lait fourni par des femmes de toutes conditions. La création de ce dépôt a posé des problèmes qui autrefois ne présentaient pas une grande impörtance, mais qui aujourd'hui sont venus au premier plan et qui doivent être résolus si ce genre d'organisation est appelé à subsister et même à se développer.

Il n'est plus possible en effet aujourd'hui de demander à des mères de se laisser prélever leur lait superflu, dans les établissements, sous un contrôle rigoureux. Le lait est en effet recueilli au domicile de la mère et par ses propres soins ; il faut donc pouvoir, au moment où il arrive au dépôt central, en contrôler la qualité. En particulier, le problème s'est posé en vue de lutter contre les fraudes possibles, de rechercher l'addition de lait de vache au lait de femme, recherche qui, jusqu'ici, avait fait l'objet de très peu de travaux.

Nous citerons parmi les publications faites sur ce sujet l'étude de KAPELLER [1], qui, alors qu'il était directeur du Service de vérification des produits alimentaires à Madgebourg, élabora une méthode réfractométrique pour permettre de rechercher l'addition de lait de vache au lait de femme. Cette méthode fut appliquée en particulier au premier dépôt central de lait de femme que je fondai dans cette ville en 1919.

Par contre, les autres méthodes de recherche du lait de vache dans le lait de femme, que l'on trouve décrites dans la littérature, reposent surtout sur des réactions colorées qui, malheureusement, ne donnent pas une sécurité suffisante pour un contrôle pratique. Nous

(1) Deutsche Medizinische Wochenschrift, 1938, N. 9, p. 297. Traduction G. Gর́nIN. 
citerons, par exemple, une réaction colorée qui a, à nouveau, été proposée au cours de ces derniers mois, et qui repose sur l'emploi d'une solution à $0,25 \%$ de bleu de Nil. On a examiné avec ce réactif 55 échantillons de lait de femme parmi lesquels il s'en trouvait 15 qui étaient des échantillons falsifiés par addition de 15 ou de $10 \%$ de lait de vache. L'emploi de ce réactif coloré a permis de déceler 10 des échantillons falsifiés. Par contre, 5 ont pu échapper à l'examen de contrôle, et parmi ces 5 , il s'en trouvait 3 qui renfermaient $15 \%$ de lait de vache. Par contre, l'emploi du réactif coloré a conduit à considérer eomme falsifiés 3 échantillons de lait de femme parfaitement purs. Si d'ailleurs, au lieu de procéder à ce contrôle dès que le lait est recueilli, on y procède après un délai de 24 heures, on constate que le nombre des indications erronées devient encore plus grand. J. BAUER, qui a conseillé l'emploi de cette méthode colorimétrique, indique qu'elle n'est applicable que pour déceler les additions de lait de vache supérieures à $30 \%$.

C'est la raison pour laquelle, dès 1920, j'avais essayé d'utiliser dans le dépôt central de lait de femme les méthodes biologiques en en rendant l'exécution suffisamment simple. KIonka, d'Iéna, avait bien voulu m'aider très aimablement dans ces recherches; malheureusement l'inflation de la monnaie allemande mit alors fin prématurément à ces travaux et le dépôt central de lait de femme de Magdebourg disparut.

Au printemps 1927, travaillant dans les laboratoires de la nouvelle installation du dépôt central d'Erfurt, je réussis à mettre au point une méthode de différenciation pratique et simple du lait de femme et du lait de vache, méthode qui a été décrite pour la première fois par KAYSER, à la séance de I'Association pour l'avancement de tout ce qui concerne l'accouchement (Bonn, juillet 1927), et cette méthode, jointe à l'essai indiqué par Margarete ZimmenMANN [2], permettait de déceler des additions de $10 \%$ de lait de vache au lait de femme. Ce premier succès permit de répondre aux exigences de la pratique du moment.

Toutefois, avec le développement de l'activité du dépôt central de lait de femme, cette méthode, malgré sa simplicité, se montrait relativement longue. Il fallait, pour obtenir des résultats exacts en appliquant les méthodes que nous venons d'indiquer, prélever un échantillon sur chaque livraison de lait provenant de chacune des nourrices attachées à l'établissement, comparer cet échantillon à celui d'un lait fourni par la même femme, mais en présence d'un eontrôleur (eet échantillon était appelé lait de contrôle) et il fallait également le comparer à un autre échantillon préparé en ajoutant au lait de contrôle $10 \%$ de lait de vache. (Ce dernier échantillon étant appelé falsification de contrôle.) 
Lorsque le nombre des nourrices attachées à l'établissement n'était pas trop élevé et tant que le nombre des auxiliaires fut suffisant, il fut toujours possible de recueillir les échantillons de lait de contrôle et de préparer les différents échantillons servant aux essais, mais la complexité de cette méthode se faisait déjà sentir en 1932 et, dès 1933, j'entrepris de nouveaux essais reposant sur l'emploi d'échantillons sérologiques.

Les essais devaient porter tout d'abord sur l'emploi du sérum décrit par J. BAUER [3], sérum qu'on espérait pouvoir obtenir de la firme allemande "Sächsische Serumwerk» à Dresde. Malheureusement, cette firme ne fabriquait pas ce sérum. SAUER, du Service public de contrôle d'Erfurt, essaya de préparer ce sérum d'après les indications de BAUER, mais n'y parvint pas.

C'est alors que j'entrepris l'étude de la méthode de Koschuscharoff décrite par JORDANOFF [4] en 1932 et avec laquelle cet auteur était parvenu à déceler de très légères falsifications de différentes sortes de lait d'animaux. Après beaucoup d'essais inutiles pour obtenir ce sérum en Allemagne, l'Institut de bactériologie vétérinaire de Sofia me fit parvenir, sur ma demande, du lactotest de lait de vache et je dois remercier bien sincèrement le Directeur de l'Institut pour cet envoi.

Les recherches portèrent sur plus de 70 essais particuliers, et bien que le nombre de ces essais ne soit pas très considérable, la conclusion de cette première série d'études fut que l'emploi de ce lactotest permettait, avec une certitude parfaite, de déceler la présence dans le lait de femme de faibles quantités de lait de vache.

Nous nous attachions surtout dans ces études à examiner le lait du plus grand nombre possible de sujets et à des périodes différentes de lactation variant de 8 jours à 12 mois. De même, nous essayions les différentes portions de lait fournies par les divers prélèvements d'une même journée. Enfin, comme la pratique avait montré que les fraudes portaient le plus souvent sur des additions de lait de vache en proportion supérieure à $10 \%$, la plupart des essais furent effectués sur des mélanges contenant $10 \%$ de lait de vache. Mais nous effectuâmes aussi des essais avec des additions plus faibles $(5 \%$ et $2 \%$ de lait de vache; des additions encore plus faibles ne se firent pas). Même les additions aussi faibles restaient encore nettement et incontestablement décelables.

Comme lait de vache ajouté au lait de femme, nous avons employé soit le lait de vache tel qu'il est vendu dans le commerce, soit des échantillons de lait que l'on faisait au préalable bouillir. Les additions de lait de vache se faisaient soit le jour même de l'essai, soit le jour précédant l'essai, afin de reproduire exactement les conditions de la fraude telle qu'elle peut se produire dans la pratique. Dans certains cas, les échantillons contenant/des additions de lait 
de vache furent aussi transportés à travers la ville dans l'auto du commerce pour imiter toutes les possibilités arrivant dans la pratique. Les essais portèrent également sur des échantillons de lait de femme dit lait de foie. Ces laits sont ceux qui sont fournis par une nourrice ayant peu de temps auparavant consommé du foie et ils montrent une luminescence jaune [5 et 6] analogue à celle du lait de vache. Or, ce lait de foie donna à la réaction de Koschuscharoff un résultat négatif, de même que le lait fourni par une femme ayant absorbé auparavant 1 litre et demi de lait de vache. Ce lait de femme présente une intensité luminescente qui correspond à celle d'un lait de femme ordinaire additionné de $25 \%$ de lait de vache.

Nous n'avons pas tenu compte des essais portant sur des échantillons de lait de femme falsifiés par addition de lait de vache stérilisé, car ces essais ont été faits en très faible nombre et ils ont donné des résultats divergents. D'ailleurs, dans ce cas particulier, il n'y aurait pas un grave inconvénient à ce que la méthode de Koschuscharoff ne donne pas de résultat exact, car on ne conçoit pas qu'une femme puisse disposer de lait stérilisé pour frauder le lait qu'elle fournit.

On a également constaté qu'en conservant pendant 6 jours à l'état non bouilli un mélange de lait de femme et de lait de vache, on obtient un mélange qui donne un résultat négatif à la réaction. Dans ce cas le degré d'acidité s'élevait à $8^{\circ} \mathrm{S}$. $-\mathrm{H}$, et le lait présentait un fort dépôt à la centrifugation.

Les essais ont été effectués en suivant le mode opératoire décrit par IWANSK. On prélève $5 \mathrm{~cm}^{3}$ de la matière que l'on a à examiner et qui est complètement dégraissée par centrifugation. Une goutte du produit dégraissé est alors mélangée avec l'aide d'une baguette en verre avec 4 fois son poids de lactotest en opérant sur un porteobjet de microscope. En opérant avec précaution et si le lait de femme est additionné de lait de vache, la réaction apparaît positive, après environ une demi-minute. Le liquide qui est trouble au début s'éclaircit et, dans les parties périphériques, la coagulation apparaît très nette. Au contraire, avec du lait de femme pur, la goutte reste uniformément trouble et il apparaît au centre un nuage blanchâtre.

Cette méthode présente pour un dépôt central de lait de femme les grands avantages suivants :

$1^{0}$ Il n'est plus nécessaire de se procurer du lait de contrôle;

$2^{\circ}$ Comme les plus petites falsifications sont décelables, il est possible d'opérer sur un échantillon obtenu par mélange de difrérentes portions de lait fournies par une donneuse individuelle;

$3^{\circ}$ Les dépenses de réactif sont réduites ;

4: La réaction est rapide. 
Actuellement, une difficulté subsiste; elle réside dans la difficulté de se procurer le sérum nécessaire aux réactions. Comme le procédé Koschuscharoff est breveté, il serait souhaitable que l'Allemagne obtienne une licence de fabrication, car il n'est pas possible d'envisager que Sofia fournisse régulièrement ce sérum.

\title{
RÉFÉRENCES
}

[1] Kapeller et A. Gotthried. M. m. W., 9, VII, 1920.

[2] M. Zimmermann. Z. Kinderhk, 1928, p. 310.

[3] J. BAUER. Methodik der Biologisehen Milehuntersuchung.

[4] JoRdanoff. Z. Fleiseh- u. Milchhyg., 1, V, 1932.

[5] Marie-Elise KAyser. Welcher Leberbestandteil geht in die Frauenmilch über. Deutsehe Medizinische Wochenschrift, n 4, 1937, p. 136.

[6] Marie-Elise Kayser. Leberbestandteile in der Frauenmilch. Deutsche Medizinische Wochenschrift, no 18, 1937, p. 712.

\section{REVUE \\ LES CONDITIONS DU MARCHÉ MONDIAL DE LA CASÉINE A LA FIN DE 1937}

\author{
par \\ G. GÉNIN \\ Ingénieur E.P.C.
}

Le $D^{r}$ James Rubinfeld a publié il y a quelques semaines dans la revue "British Plastics» une étude sur les conditions du marché mondial de la caséine, qu'il nous a paru intéressant de faire connaître à nos lecteurs, étant donné l'importance que présente pour la France la connaissance des tendances sur le marché de la caséine.

A la fin de 1937, les cours de la caséine argentine s'établissaient à Londres, pour la marchandise cif à 49 livres par tonne, plus les droits de douane. Vers le milieu de mars de la même année, les cours s'élevaient jusqu'à 59 livres, mais à partir de ce moment, la tendance à la baisse devait apparaître, le prix de la caséine s'abaissait à 52 livres vers la fin d'avril pour rester à ce niveau jusque vers le milieu d'octobre et s'abaisser ensuite à 48 livres. On voit done que l'élévation des cours enregistrés vers le début de l'année était complètement annulée et que les craintes qu'on avait eues à un moment donné de voir les cours s'envoler à la suite de la reprise économique dans certains pays ne s'étaient pas réalisées.

Depuis la fin de 1937, les cours n'ont d'ailleurs fait que continuer leur tendance à la baisse. En novembre 1937, ils étaient de 44 livres par tonne et de 39 seulement vers le milieu de décembre. On voit done que ce dernier cours ne diffère pas beaucoup de la valeur enregistrée en octobre 1935, en pleine période de crise et au 